\title{
Less Is More: Semantic Information Survives Interocular Suppression When Attention Is Diverted
}

\author{
Kangyong Eo, ${ }^{1}$ Oakyoon Cha, ${ }^{1}$ Sang Chul Chong, ${ }^{1,2}$ and Min-Suk Kang ${ }^{3,4}$ \\ ${ }^{1}$ Graduate Program in Cognitive Science and ${ }^{2}$ Department of Psychology, Yonsei University, Seoul 03722, Republic of Korea, ${ }^{3}$ Center for Neuroscience \\ Imaging Research, Institute for Basic Science, Suwon 16419, Republic of Korea, and ${ }^{4}$ Department of Psychology, Sungkyunkwan University, Seoul 03063 , \\ Republic of Korea
}

The extent of unconscious semantic processing has been debated. It is well established that semantic information is registered in the absence of awareness induced by inattention. However, it has been debated whether semantic information of invisible stimuli is processed during interocular suppression, a procedure that renders one eye's view invisible by presenting a dissimilar stimulus to the other eye. Inspired by recent evidence demonstrating that reduced attention attenuates interocular suppression, we tested a counterintuitive hypothesis that attention withdrawn from the suppressed target location facilitates semantic processing in the absence of awareness induced by interocular suppression. We obtained an electrophysiological marker of semantic processing (N400 component) while human participants' spatial attention was being manipulated with a cueing paradigm during interocular suppression. We found that N400 modulation was absent when participants' attention was directed to the target location, but present when diverted elsewhere. In addition, the correlation analysis across participants indicated that the N400 amplitude was reduced with more attention being directed to the target location. Together, these results indicate that inattention attenuates interocular suppression and thereby makes semantic processing available unconsciously, reconciling conflicting evidence in the literature. We discuss a tight link among interocular suppression, attention, and conscious awareness.

Key words: attention; consciousness; event-related potential; interocular suppression

\section{Significance Statement}

Interocular suppression offers a powerful means of studying the extent of unconscious processing by rendering a salient stimulus presented to one eye invisible. Here, we provide evidence that attention is a determining factor for unconscious semantic processing. An electrophysiological marker for semantic processing (N400 component) was present when attention was diverted away from the suppressed stimulus but absent when attention was directed to that stimulus, indicating that inattention facilitates unconscious semantic processing during the interocular suppression. Although contrary to the common sense assumption that attention facilitates information processing, this result is in accordance with recent studies showing that attention modulates interocular suppression but is not necessary for semantic processing. Our finding reconciles the conflicting evidence and advances theories of consciousness.

\section{Introduction}

Interocular suppression has been a pivotal tool for scientific studies of visual awareness because our conscious visual awareness is

\footnotetext{
Received Aug. 8, 2015; revised April 14, 2016; accepted April 16, 2016.

Author contributions: S.C.C. and M.-S.K. designed research; K.E. and O.C. performed research; K.E. and 0.C. analyzed data; K.E., O.C., S.C.C., and M.-S.K. wrote the paper.

This work was supported by the National Research Foundation of Korea Grant NRF-2011-0025005 (funded by the Korean government) to S.C.C. and NRF-2013S1A5A8025812 to M.-S.K. We thank Randolph Blake and Jan Brascamp for their helpful comments.

The authors declare no competing financial interests.

Correspondence should be addressed to Dr. Min-Suk Kang, IBS Center for Neuroscience Imaging Research, N Center, Sungkyunkwan University, Seobu-ro 2066, Jangan-gu, Suwon 16419, Republic of Korea. E-mail: kangminsuk@skku.edu.

DOI:10.1523/JNEUROSCI.3018-15.2016

Copyright $\odot 2016$ the authors $\quad 0270-6474 / 16 / 365489-09 \$ 15.00 / 0$
}

dissociated from physical inputs, such that even a salient stimulus presented to one eye is rendered invisible when a competing, rival stimulus is presented to the other eye (Kim and Blake, 2005). With this form of dissociation, scientists have asked to what extent visual stimuli in the suppression phase are processed in the absence of awareness. It is now well established that basic features (e.g., stimulus orientation and motion direction) are registered without awareness (Wiesenfelder and Blake, 1990; Blake et al., 2006), but it has been intensely debated whether our brain can extract semantic information of invisible words during interocular suppression (Lin and He, 2009; Gayet et al., 2014; Sterzer et al., 2014; Yang et al., 2014).

There is conflicting evidence for unconscious semantic analysis for invisible stimuli during interocular suppression. For instance, 


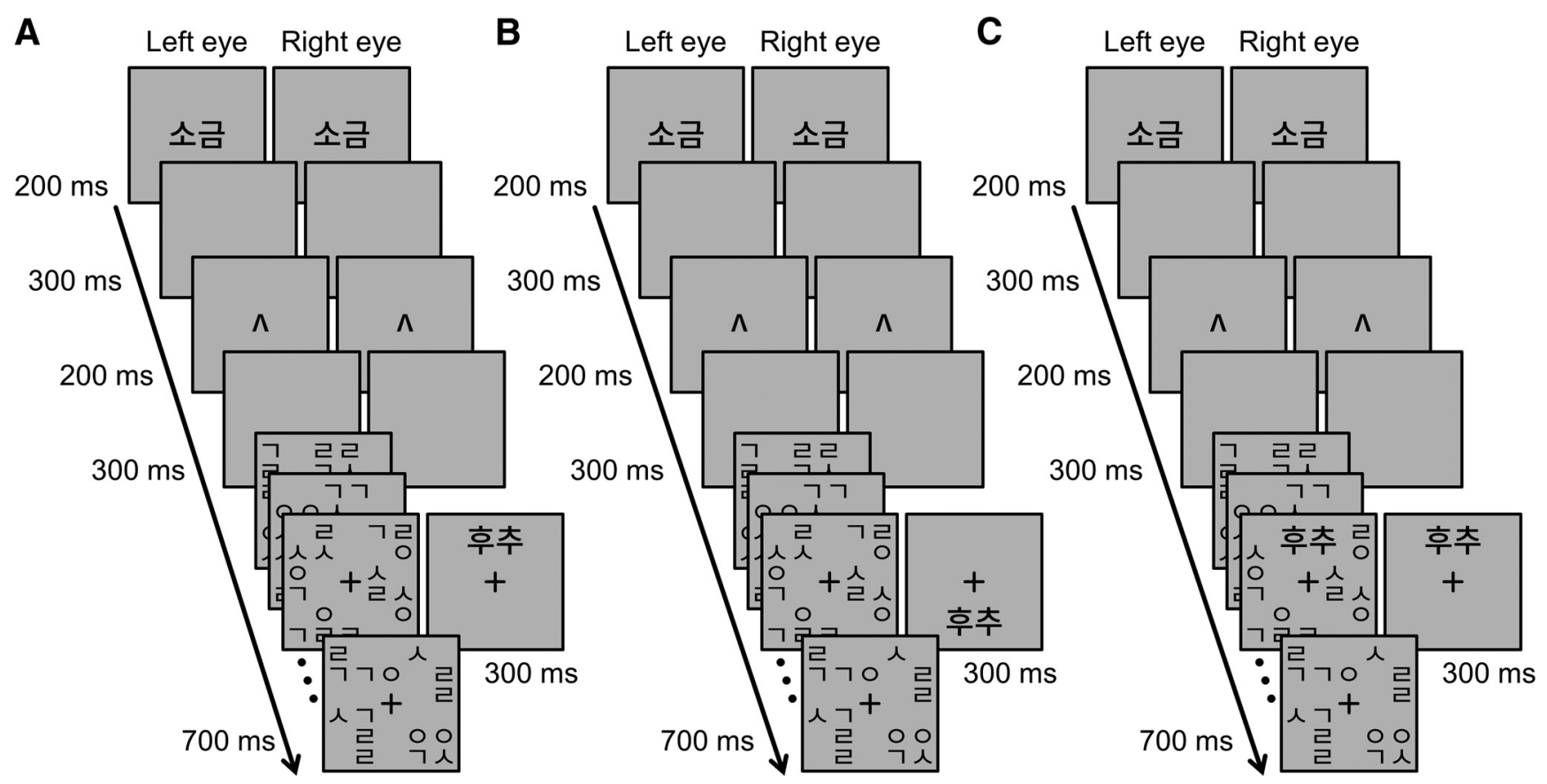

Figure 1. The experimental procedure of the congruent $(\boldsymbol{A})$, incongruent $(\boldsymbol{B})$, and visible $(\boldsymbol{C})$ conditions. A prime word $(200 \mathrm{~ms})$, arrow cue ( $200 \mathrm{~ms})$, and CFS stimuli with a target word are presented in sequence, separated by two $300 \mathrm{~ms}$ blank screen intervals. The CFS stimuli, composed of seven different frames of Korean letters, are presented to one eye for $700 \mathrm{~ms}$, while the target word is presented to the other eye from the third to fifth frames. $\boldsymbol{A}$, In the congruent condition, the target word is presented to the cued location. $\boldsymbol{B}$, In the incongruent condition, the target word is presented opposite to the cued location. $C$, In the visible condition, the target word is presented to the cued location of both eyes.

prior works have shown that semantically relevant stimuli (e.g., word and mathematical notation) break suppression faster than irrelevant stimuli during continuous flash suppression (CFS), a form of interocular suppression, which is construed as evidence for unconscious semantic processing (Costello et al., 2009; Bahrami et al., 2010; Yang and Yeh, 2011; Sklar et al., 2012; Zabelina et al., 2013). However, other empirical studies fail to demonstrate neural and psychophysical signatures of semantic processing when the suppressed stimuli are invisible (Zimba and Blake, 1983; Blake, 1988; Kang et al., 2011; Hesselmann et al., 2015).

We reasoned that attention is a determining factor modulating unconscious semantic processing during interocular suppression based on the following lines of research. First, attention is not necessary for semantic processing: unattended semantic information is processed in the absence of awareness during attentional blink and visual search (Luck et al., 1996; Vogel et al., 2005; Giesbrecht et al., 2007). Second, more important for the present study, reduced attention attenuates the strength of interocular suppression (Bahrami et al., 2007; Zhang et al., 2011; Brascamp and Blake, 2012; Ling and Blake, 2012). In particular, participants can detect probes presented to the suppressed eye at weaker contrast levels when attention is withdrawn from the rival stimuli, compared with the contrast levels of the probes when attention is directed to them (Ling and Blake, 2012; Stein et al., 2012). Psychophysical and electrophysiological signatures of perceptual alternations are also abolished in the absence of attention (Zhang et al., 2011; Brascamp and Blake, 2012). Together, we hypothesized that inattention facilitates unconscious semantic processing because reduced attention attenuates rivalry suppression; thus, semantic information becomes available while participants are not aware of the stimulus due to inattention.

In the present study, we presented prime and target words in sequence and participants were asked to determine whether those words were semantically related (Fig. 1). Semantic processing in the absence of awareness was probed using the $\mathrm{N} 400$ component, which is a sensitive event-related potential (ERP) distinguishing the meaning of words: ERPs elicited by unrelated targets are more negatively deflected than related targets $\sim 400 \mathrm{~ms}$ after stimulus of the target onset (Kutas and Hillyard, 1980; Kutas and Federmeier, 2011). To manipulate participants' attention, we adopted a cueing paradigm such that the target word was presented at either a cued location (Fig. $1 A$ ) or its opposite side (Fig. 1B). Because the target word was suppressed because of CFS (Tsuchiya and Koch, 2005), participants were not aware of its meaning. If inattention does facilitate unconscious semantic processing, the N400 modulation should be absent when participants' attention is directed toward the target location (congruent condition; Fig. 1A) but should emerge when their attention is diverted outside the target location (incongruent condition; Fig. 1B).

\section{Materials and Methods}

Overview. Participants performed a semantic judgment task for the sequentially presented prime and target words in both Experiments 1 and 2. The target word was rendered invisible with interocular suppression. In Experiment 1, we recorded ERPs to probe unconscious semantic processing and then the visibility of the target word was measured in a separate session. In Experiment 2, we conducted a behavioral experiment to examine the influence of stimulus visibility on semantic judgment performance directly. In each trial, participants judged semantic relatedness between the prime and target words and then reported the target location.

Participants. Sixty-eight volunteers (40 for Experiment 1 and 28 for Experiment 2) with normal or corrected-to-normal vision (37 female, age range 19-34 years, mean $24.1 \pm 2.8$ years) participated in the experiments, and they provided written informed consent. The study was approved by the Institutional Review Board of Yonsei University. All participants were naive to the purpose of the experiment.

In Experiment 1, data from 24 participants were analyzed after removing data from 16 participants. We applied the following criteria in sequence. First, 2 participants' data were excluded because they did not 
follow the instruction: their semantic judgment performances in the visible condition were $39.8 \%$ and $50.0 \%$. Second, we excluded 9 participants' data due to recording related noise. Eight participants' data were excluded because trials containing oculomotor artifacts and muscle noise exceeded $50 \%$ of trials $(56 \%-78 \%)$. We set the $50 \%$ criterion because this procedure resulted in 27 trials per condition on average, providing a minimum number of trials for the ERP component of interest. We also excluded one dataset because of fluctuations in impedance level. Third, previous studies indicate that the potency of CFS is limited (e.g., Yang and Blake, 2012); thus, it is important to establish that the target words were not consciously available during the experiment. We excluded participants' data if the target words were consciously available based on independent measures of stimulus visibility, and not based on either their ERPs or semantic judgment performance. Visibility of the target word was probed using a word recognition task and a location judgment task as they provide an upper and a lower benchmark for the target visibility, respectively. We excluded participants' data if the word recognition performance exceeded $63 \%$ (the upper limit of the $95 \%$ confidence interval from a binomial distribution if performance is at chance, $p=0.5, N=60$ ). Two participants' data were excluded ( $68 \%$ and $83 \%)$ based on this criterion. In addition, we excluded 2 participants' data based on their location judgment performance ( $95 \%$ and $97.5 \%$ ) if the participants could correctly detect the target location $>90 \%$. We were concerned that the cue could have been ineffective at directing participants' attention in the main experiment for frequent occurrence of the target at the uncued location, although they could not recognize it. The semantic judgment performance of these two participants was $64.8 \%$ and $50 \%$ in the congruent condition, respectively. Considering that the upper limit of the $95 \%$ confidence interval of the semantic judgment task (a binomial distribution with $p=0.5$ and $N=108$ ) is $60 \%$, one might argue that we should include one participant's data, showing $50 \%$ accuracy in the semantic judgment task, even with high location judgment performance $(97.5 \%)$, but inclusion of the data did not change the pattern of our results.

In Experiment 2, 28 participants' data were collected. We excluded 6 participants' data that showed an average location judgment performance of $90 \%$ for the congruent and incongruent conditions to make it comparable with Experiment 1 (92.7\%-100\%).

Apparatus. The experiment was controlled by a program created using the Psychophysics Toolbox for MATLAB running on a Pentium dualcore computer (Brainard, 1997; Pelli, 1997). Stimuli were presented on a gamma-corrected HP p1230 CRT 21 in. monitor $(85 \mathrm{~Hz}$ in frame refresh rate, $60 \mathrm{~cm}$ viewing distance) in a dark room. Rival stimuli were presented to each eye using red-green anaglyph glasses (Almeida et al., 2008; Kang et al., 2011).

Experiment 1: stimuli. For interocular suppression, we created dynamically changing noise patterns. Each pattern was created with $\sim 40 \mathrm{Ko}-$ rean letters consisting of four consonants that randomly filled an $11 \times 11$ $\operatorname{grid}\left(7.37^{\circ} \times 7.37^{\circ}\right)$ surrounded by a black frame. The size of each syllable was $\sim 0.67^{\circ} \times 0.67^{\circ}$.

The semantically related prime and target pairs were prepared as follows. We created 324 pairs of semantically related Korean words (Park, 2004), and the word lengths of the prime and target were matched for each pair (nine pairs of single, 282 pairs of double, 27 pairs of triple, and six pairs of quadruple syllable words). The word pairs were distributed among six sets with their statistical properties, such as frequencies of word length, being as close as possible. Half of the six sets was used to create related pairs and the other half was used to create unrelated pairs by swapping target words (Vogel et al., 2005) so that two sets were tested for three different conditions (congruent, incongruent, and visible). The six sets were assigned across all participants so that each participant was given a unique combination of word sets for three conditions $\times$ two pair types (related vs unrelated).

Procedure and design. The experiment consisted of three separate phases: a brief calibration session, a main experimental session with EEG recording, and a confirmation session. In the calibration session, the Weber contrast (difference in luminance between the target and background divided by the background luminance) of target words was determined as either $40 \%$ or $60 \%$. After dark adaptation, a random word that was not used in the main experiment was presented above or below the fixation cross with CFS. At first, we presented $60 \%$ contrast stimuli; and if participants could easily detect the target with that contrast based on verbal communication, we lowered the contrast by $20 \%$. We did not decrease the contrast level any further based on a pilot experiment in which it was difficult to recognize the target with the $20 \%$ contrast level, even in the visible condition where the target was embedded with the full contrast CFS stimuli (visible condition).

Figure 1 illustrates the stimulus sequences of the three different conditions of the main experiment. Briefly, each trial began with a black fixation cross for $600 \mathrm{~ms}$. A black rectangular frame was presented to both eyes to promote binocular alignment throughout the trial. A prime word was presented to both eyes for $200 \mathrm{~ms}$, followed by a blank screen for $300 \mathrm{~ms}$, and then an arrow cue, directed either up or down, was presented for $200 \mathrm{~ms}$. After a blank frame was presented for $300 \mathrm{~ms}$, the CFS was presented (Korean letters) to one eye for $700 \mathrm{~ms}$ with seven different frames lasting $100 \mathrm{~ms}$ each. A target word was turned on abruptly $300 \mathrm{~ms}$ after the onset of the CFS stimuli above or below the fixation cross and then abruptly turned off $300 \mathrm{~ms}$ after its presentation. The target word was presented in the cued location in the congruent (Fig. 1A) and visible (Fig. 1C) conditions. In the incongruent condition (Fig. 1B), the target word was presented opposite to the cued location. The target word was presented to the eye opposite to the CFS stimuli in the congruent and incongruent conditions, but to both eyes in the visible condition. Participants were instructed to fixate their eyes on the fixation cross for $\sim 500 \mathrm{~ms}$ after the trial finished. Participants were instructed to press the left or right arrow key when the words were semantically related or unrelated, respectively, but they were encouraged to guess semantic relatedness if they were not confident in their answer due to CFS. A total of 324 trials, with an equal number of the three conditions (congruent, incongruent, and visible) $\times$ pair type (related vs unrelated), were administered in a pseudorandom order. Participants were given a break every 24 trials.

We used two tasks to independently confirm the visibility of the target word following the main experiment: location judgment and word recognition tasks. The procedure for the location judgment task was the same as the main experiment, except that a prime word and a cue were not presented. Instead, only the target word was dichoptically presented with CFS, either above or below the fixation cross following the $600 \mathrm{~ms}$ fixation period. Participants were instructed to determine where the target was presented with the upper and lower arrow keys. This task consisted of 40 trials and provided a lower benchmark for how much information was consciously available because participants could correctly identify the target location without recognizing its meaning. The word recognition task was identical to the location judgment task, except that participants were instructed to distinguish the target from a lure. The target word and a lure were presented in the left and right part below the fixation cross after the offset of CFS, and participants were instructed to determine which one was the target with the left and right arrow keys. This task consisted of 60 trials and provided an upper benchmark for how much information was consciously available because participants could correctly recognize the target only when the majority of the letters were visible.

Electrophysiological recording and data analysis. The EEG was recorded with $\mathrm{Ag} / \mathrm{AgCl}$ electrodes from 20 scalp locations (10-20 system: F7, F3, Fz, F4, F8, T3, C3, Cz, C4, T4, T5, P3, Pz, P4, T6, PO1, O1, Oz, O2, and PO2) using a NuAmps 40 Channel Quik-Cap (Compumedics), and the signals were amplified with a NeuroScan NuAmps Express amplifier (Compumedics). The electrodes were referenced online to the average of the left and right mastoids with their impedance kept $<5 \mathrm{~K} \Omega$. The horizontal electro-oculogram was recorded from two electrodes placed on the external canthus of each eye, and the vertical electro-oculogram (VEOG) was recorded from two electrodes placed above and below the left eye. A $0.05-100 \mathrm{~Hz}$ bandpass filter was applied online with $250 \mathrm{~Hz}$ sampling frequency. Trials with eye movements, blinks, muscle noise, or amplifier saturation were defined with a standard procedure (Luck, 2014 ), and then rejected before averaging ( $12 \%-41 \%$ of trials). A $30 \mathrm{~Hz}$ low-pass filter was applied to the ERPs to create Figure 2, but statistical analyses were performed on the unfiltered data.

The ERPs, EEGs, and EOGs were obtained from -100 to $800 \mathrm{~ms}$ with respect to the onset of the target. The baseline was corrected using the -100 to $0 \mathrm{~ms}$ interval before the onset of the target. The N400 amplitudes 

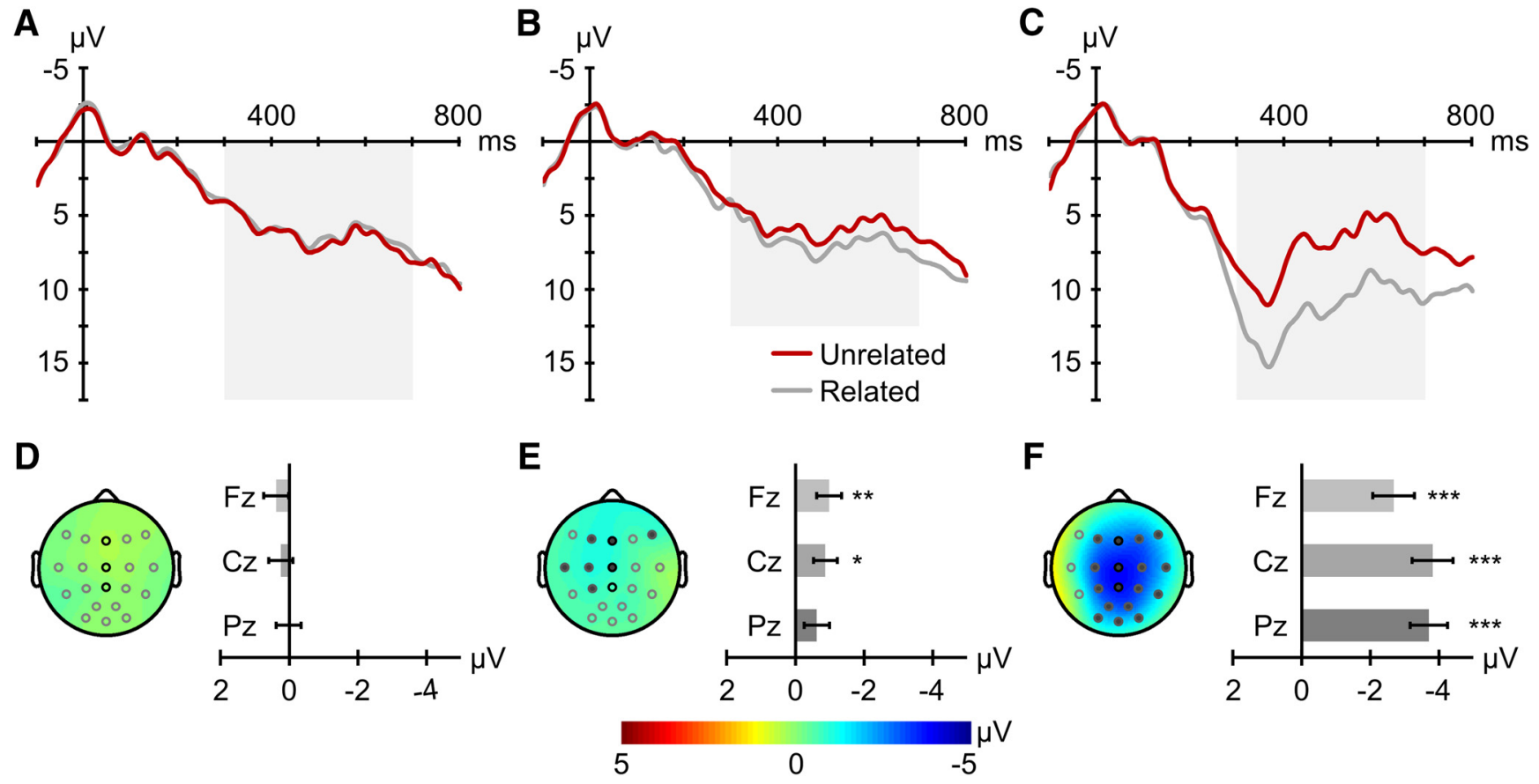

Figure 2. ERP results $(N=24)$ obtained from the congruent $(\boldsymbol{A})$, incongruent $(\boldsymbol{B})$, and visible conditions ( $\boldsymbol{C}$. ERP waveforms obtained from the CZ electrode are locked with the onset of the target.

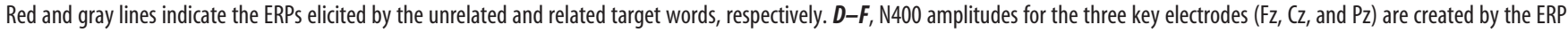
difference (unrelated - related) within the 300-700 ms post-target word onset temporal window. Error bars indicate SEM. ${ }^{*} p<0.05$ ( $t$ test). ${ }^{* *} p<0.01$ ( $t$ test). ${ }^{* * *} p<0.001$ ( $t$ test). N400 amplitudes from all electrodes are topographically plotted, with a significant modulation for all levels $(p<0.05)$ marked with a filled circle at the corresponding electrode position.

in the congruent, incongruent, and visible conditions were obtained within the 300-700 ms temporal window post-target word onset by subtracting the ERP waveforms elicited by the unrelated target word from the ERP waveforms elicited by the related target word. The VEOG amplitude was also calculated within the same temporal window of the N400. Because the N400 is maximal over centroparietal sites (Swaab et al., 2012), the three key electrodes (Fz, $\mathrm{Cz}$, and $\mathrm{Pz})$ were selected as representatives of the N400 amplitudes. However, we also present the voltage differences over the entire electrodes topographically using EEGLAB (Delorme and Makeig, 2004). The correlation between the N400 amplitude and location judgment performance was analyzed in both the congruent and incongruent conditions to examine the degree of association between inattention and N400 amplitude in each condition.

Experiment 2. Participants performed a semantic judgment task, and then a location judgment task in each trial. The semantic judgment task of Experiment 2 was similar to that of Experiment 1, except for the following: we used 144 pairs of double syllable words and assigned 24 trials for each condition (visible, congruent, and incongruent $\times$ related and unrelated). The location judgment task followed immediately, with participants having to indicate the location of the target word with the upper and lower arrow keys.

\section{Results}

\section{Experiment 1: behavioral and} electrophysiological experiment

Visible condition

In the visible condition, both prime and target words were presented to both eyes, and the target was always presented at the cued location (Fig. 1C). Participants could correctly judge semantic relatedness $(92.63 \pm 3.54 \%)$. The visible condition was designed to promote cue validity and participants' motivation, without which participants would have to determine semantic relatedness for invisible stimuli for the majority of trials (Pratte and Rouder, 2009). The ERP waveforms obtained at the $\mathrm{Cz}$ electrode showed a representative N400 modulation (Fig. 2C), such that the ERPs elicited by the unrelated target produced a more negative potential than the related target. The N400 amplitude from the three key electrodes (Fz: $t_{(23)}=4.40, p<0.001 ; \mathrm{Cz}$ : $t_{(23)}=6.40, p<0.001$; and Pz: $\left.t_{(23)}=6.82, p<0.001\right)$ and its topographical distribution obtained within the $300-700 \mathrm{~ms}$ posttarget word onset conformed to known N400 characteristics, with its peak at the $\mathrm{Cz}$ electrode (Fig. $2 F$ ).

Inattention facilitates unconscious semantic processing during CFS Participants occasionally extracted the meaning of the target word presented with CFS in the congruent condition (53.13 \pm $\left.3.89 \% ; t_{(23)}=3.94, p=0.001\right)$ but not in the incongruent condition $\left(50.42 \pm 2.06 \% ; t_{(23)}=1.01, p=0.324\right)$, which was near the chance level semantic judgment performance. We also confirmed that participants could not recognize the target words based on a word recognition task followed by the semantic judgment task $\left(50.97 \pm 6.81 \% ; t_{(23)}=0.70, p=0.491\right)$. In the word recognition task, the target was presented with the CFS just as in the main experiment and participants had to discriminate the target word against a lure. These results established that participants were not aware of the suppressed information during the main experiment, especially in the incongruent condition.

Our main question is whether semantic processing of the invisible target words is facilitated by directing attention outside the target location. If inattention does facilitate unconscious semantic processing, the N400 modulation should be present in the incongruent condition but absent in the congruent condition. Consistent with the hypothesis, the N400 modulation was nearly absent from all three key electrodes in the congruent condition (Fig. 2A). In contrast, the N400 modulation was evident in the incongruent condition (Fig. 2B). A three-way ANOVA with factors of congruency (congruent vs incongruent condition), pair type (related vs unrelated), and key electrode $(\mathrm{Fz}, \mathrm{Cz}$, and $\mathrm{Pz}$ ) yielded a significant interaction between congruency and pair type $\left(F_{(1,23)}=5.82, p=0.024\right)$, which means that the $\mathrm{N} 400$ 


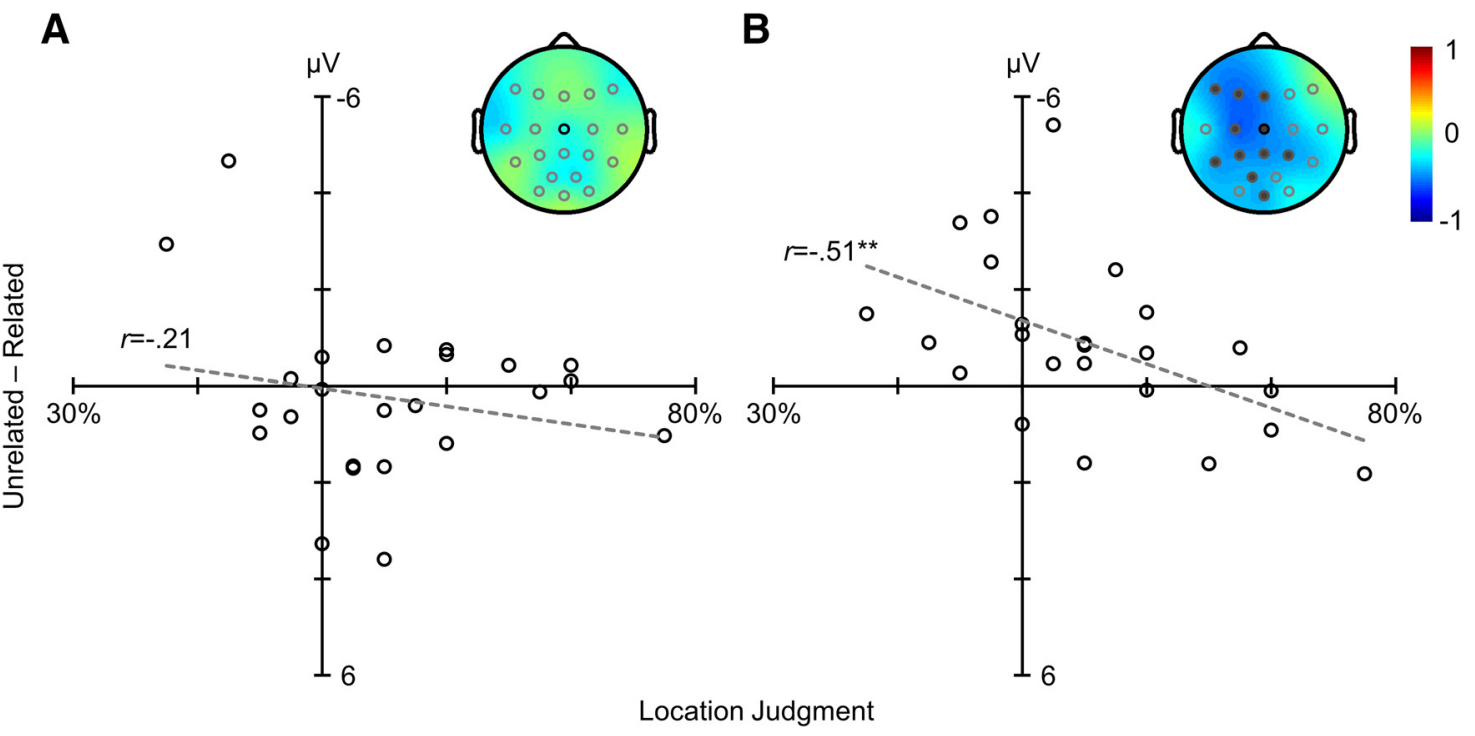

Figure 3. Correlation between $N 400$ and location judgment performance $(N=24)$. The $N 400$ amplitude (unrelated - related) is plotted as a function of location judgment performance for the congruent $(\boldsymbol{A})$ and incongruent conditions $(\boldsymbol{B})$. Dashed lines indicate linear regression lines. ${ }^{* *} p<0.05$ (correlation significance). The correlation coefficients $(r)$ from all electrodes are topographically plotted, with a significant correlation for all levels $(p<0.05)$ marked with a filled circle at the corresponding electrode position.

amplitude was significantly larger in the incongruent condition than in the congruent condition (Fig. $2 A, B$ ). A one-sample $t$ test applied to the three key electrodes showed that the N400 modulation in the incongruent condition was significant at $\mathrm{Fz}\left(t_{(23)}=\right.$ $3.04, p=0.006)$ and $\mathrm{Cz}\left(t_{(23)}=2.48, p=0.021\right)$, and marginally significant at $\mathrm{Pz}\left(t_{(23)}=1.77, p=0.090\right)$ but not in the congruent condition $\left(\mathrm{Fz}: t_{(23)}=-1.04, p=0.308\right.$; Cz: $t_{(23)}=-0.70, p=$ 0.492; Pz: $\left.t_{(23)}=-0.05, p=0.959\right)$. These results indicated that semantic processing occurred unconsciously in the incongruent condition where attention was diverted away from the target word, but the target word did not reach a level of semantic analysis in the congruent condition where attention was deployed within the target location.

\section{Stimulus visibility modulates the extent of unconscious} semantic processing

We also conducted a location judgment task with the word recognition task following the main experiment to obtain an independent measure of how much target information was consciously available during the main experiment. Location judgment performance in conjunction with the N400 amplitude obtained from the main experiment provides strong evidence for unconscious semantic processing when the participants' attention was withdrawn from the target location.

In the location judgment task, participants were instructed to determine the location of the target, which was presented either above or below the fixation cross during CFS. Location judgment performance indicated that some participants identified the target location above the chance level $\left(55.31 \pm 9.68 \%\right.$; $t_{(23)}=2.69$, $p=0.013$ ). If participants could identify the target location due to incomplete suppression, participants' attention could have been diverted to the target location especially in the incongruent condition. As a result, increased interocular suppression could disrupt unconscious semantic processing; thus, the N400 modulation should be reduced.

Consistent with this prediction, the N400 amplitude obtained in the incongruent condition was negatively correlated with location judgment performance as shown in Figure $3 B$, in which the N400 amplitude obtained from the $\mathrm{Cz}$ electrode was plotted as a function of the location judgment performance $(\mathrm{Cz}: r=$ $-0.51, p=0.012$ ). We also found similar patterns from other electrodes as one can see from the topographically distributed correlation coefficient (Fig. 3B), including two other key electrodes (Fz: $r=-0.49, p=0.014$ and Pz: $r=-0.48, p=0.017$ ). However, we did not find any correlation between the N400 amplitude and location judgment performance in the congruent condition (Fz: $r=-0.11, p=0.960$; Cz: $r=-0.21, p=0.327$; and Pz: $r=-0.23, p=0.290$; Fig. $3 A$ ). This negative correlation in the incongruent condition provided further evidence that unconscious semantic processing was facilitated when attention was fully diverted outside the target location, but interocular suppression was at full strength in the congruent condition when participants' attention was drawn to the target location and the semantic information was not available.

\section{Ruling out alternative hypotheses}

We rule out two alternative hypotheses in relation to the location judgment performance. One alternative is that an unattended stimulus in our experiment could have been consciously processed. We rule out this alternative based on two reasons. First, location judgment performance was above the chance level on average $\left(55.31 \pm 9.68 \% ; t_{(23)}=2.69, p=0.013\right)$; thus, participants could be consciously aware of the unattended target. However, if the unattended stimulus in the incongruent condition was processed consciously, semantic judgment performance should be higher in the incongruent condition than in the congruent condition, but the opposite results were obtained (congruent condition: $53.13 \pm 3.89 \%$; incongruent condition: $50.42 \pm$ $\left.2.06 \% ; t_{(23)}=3.11, p=0.005\right)$. Second, the location judgment performance did not correlate with the semantic judgment performance in both the congruent $(r=-0.09, p=0.662$; Fig. $4 A)$ and incongruent conditions $(r=-0.05, p=0.808$; Fig. $4 B)$. If incomplete suppression in the incongruent condition leads to conscious semantic processing across participants, a positive correlation should have been obtained. We will revisit this conscious semantic processing hypothesis in Experiment 2.

The other alternative posits that different patterns of eye movements explain the differences in the N400 amplitude be- 
A
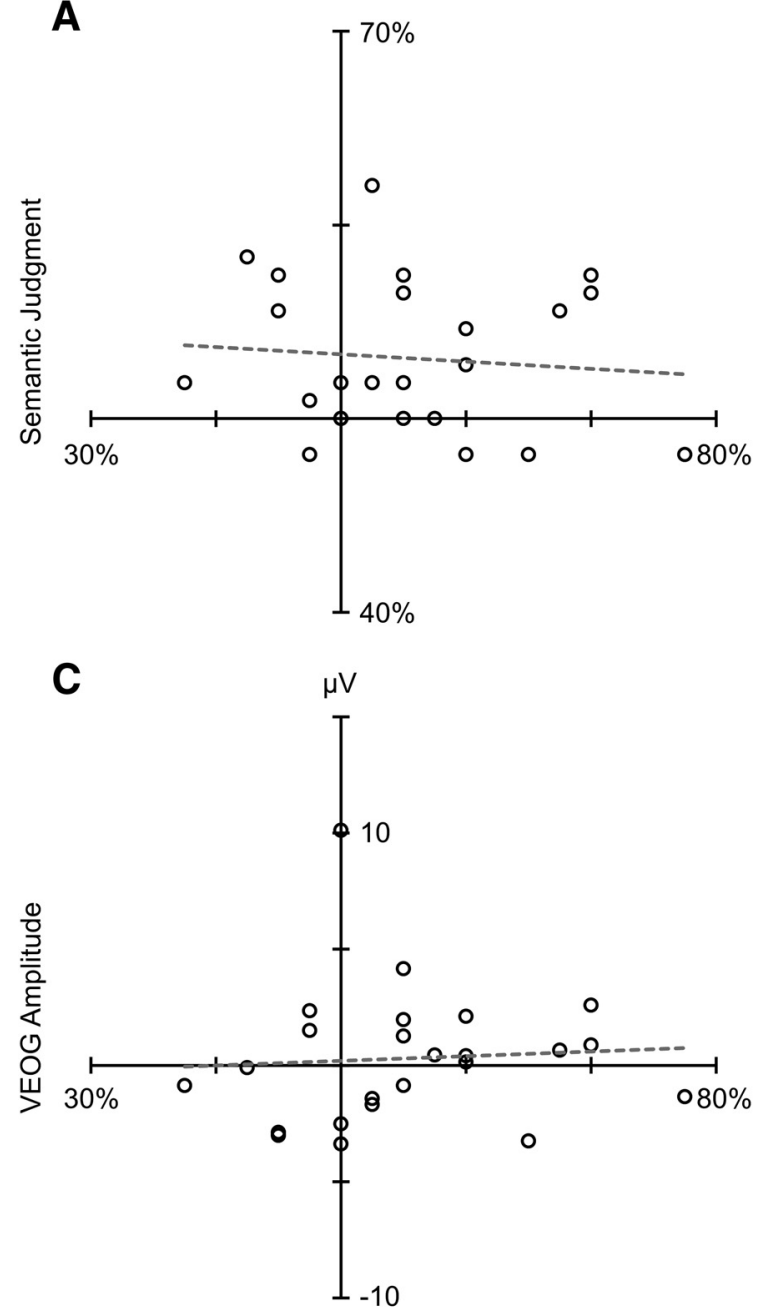

B
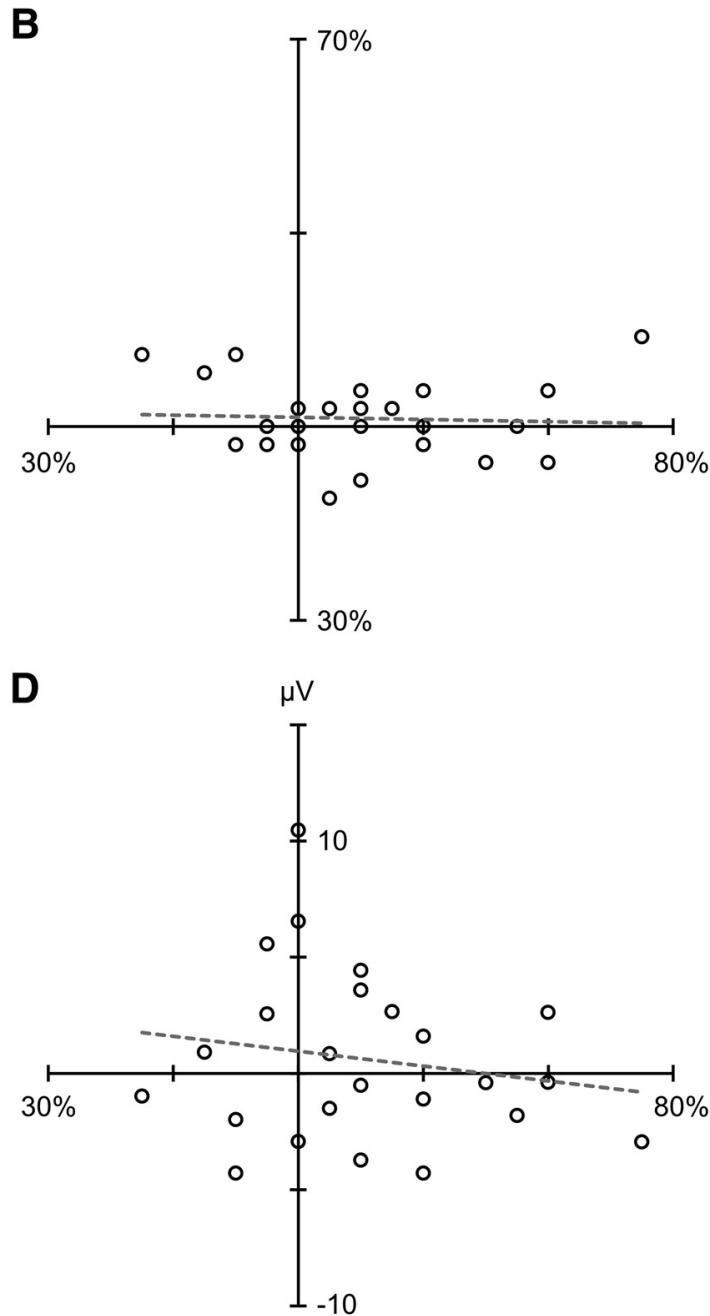

Location Judgment

Figure 4. Correlation results $(N=24)$. Semantic judgment performance is plotted as a function of location judgment performance in the congruent $(\boldsymbol{A})$ and incongruent conditions $(\boldsymbol{B})$. The VEOG amplitude is plotted as a function of location judgment performance for the congruent $(\boldsymbol{C})$ and incongruent conditions $(\boldsymbol{D})$. The VEOG amplitude is obtained from the difference between the cue conditions above and below the fixation within the $300-700 \mathrm{~ms}$ post-target word onset. Dashed lines indicate linear regression lines.

tween the congruent and incongruent conditions (Luck, 2014), but we ruled out this alternative for the following reasons. First, the related and unrelated targets were presented either above or below the fixation cross with equal probability; thus, eye movements to the target cannot explain the ERP differences associated with semantic information. In addition, the VEOGs for the pair types (related vs unrelated) within the same temporal window as the N400 amplitude were similar for both congruent and incongruent conditions (pair type: $F_{(1,23)}=0.03, p=0.868$; congruency type: $F_{(1,23)}=3.37, p=0.079$; target $\times$ congruency: $\left.F_{(1,23)}=1.74, p=0.200\right)$. Second, we also examined whether eye movements were influenced more by the cue location or target location. Because the cue and target locations coincide in the congruent condition but not in the incongruent condition, it is important to rule out whether different patterns of eye movements occurred between those two conditions. We found that the VEOG amplitudes were similar between congruent and incongruent conditions in the upper cue condition $(10.35 \mu \mathrm{V}$ and $10.62 \mu \mathrm{V}$ for the congruent and incongruent condition, respectively; $\left.t_{(23)}=-0.70, p=0.490\right)$ and in the lower cue condition (10.66 $\mu \mathrm{V}$ and $11.24 \mu \mathrm{V}$ for the congruent and incongruent condition, respectively; $\left.t_{(23)}=-1.46, p=0.157\right)$. Furthermore, Fig- ure 4, $C$ and $D$, shows the difference in VEOGs between the two cue conditions (upper cue - lower cue) as a function of location judgment performance across the participants. If the target drew the participants' eye movements due to incomplete suppression, the magnitude of eye movements should be correlated with location judgment performance in opposite directions in the congruent and incongruent conditions. However, we did not find significant correlations (Fig. $4 C$ for the congruent condition: $r=$ 0.07, $p=0.760$; Fig. $4 D$ for the incongruent condition: $r=-0.17, p=0.433)$, whereas the pattern of eye movements was similar between the congruent and incongruent conditions across participants $(r=0.63, p=0.001)$. Together, eye movements cannot explain the negative correlation between location judgment performance and the N400 amplitude obtained from the incongruent condition.

\section{Experiment 2: behavioral experiment}

We conducted a behavioral experiment as a more stringent test of whether incomplete suppression could lead to conscious semantic processing by combining the location judgment task and the semantic judgment task so that participants performed both tasks in the same trial. Experiment 2 was conducted to address the 
A

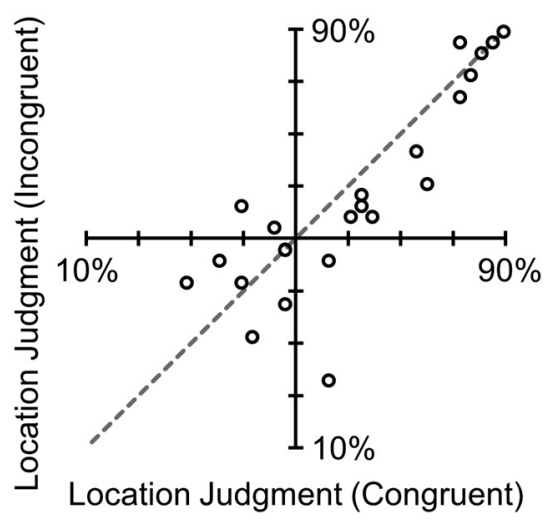

B

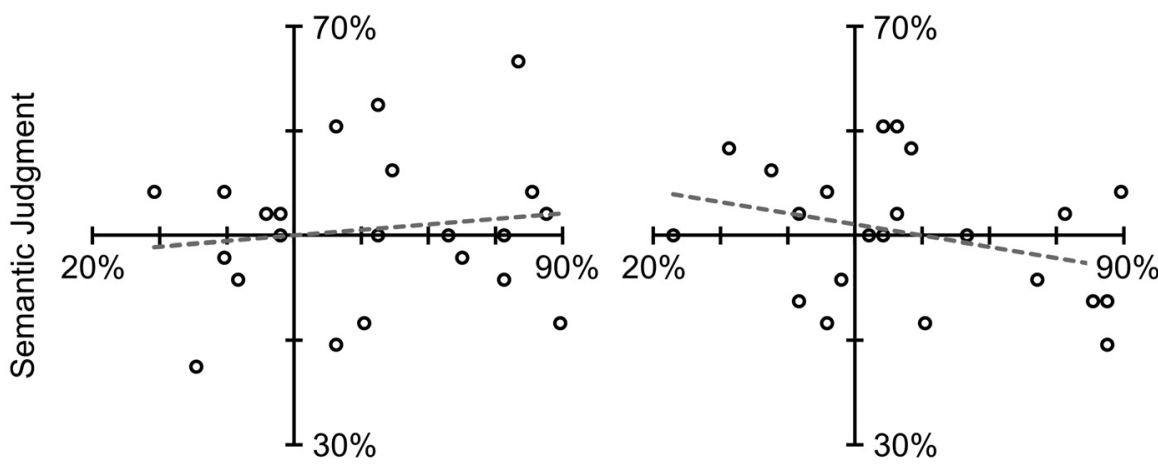

Location Judgment

Figure 5. Results of Experiment $2(N=22)$. Location judgment performance of the incongruent condition is plotted as a function of location judgment performance of the congruent condition $(\boldsymbol{A})$. The dashed line is a unity line. $\boldsymbol{B}, \boldsymbol{C}$, Semantic judgment performance is plotted as a function of location judgment performance in the congruent condition $(\boldsymbol{B})$ and incongruent condition $(\boldsymbol{C})$. Dashed lines indicate linear regression lines.

following two concerns. First, although it is common that visibility is separately measured from unconscious semantic processing in previous studies (for review, see Kouider and Dehaene, 2007), this practice has been criticized with the concern that participants can adopt different strategies or criteria across different tasks (Sterzer et al., 2014). Second, more importantly, we provided the cue to guide participants' attention and included the visible condition to promote cue validity in the semantic judgment task, but both the cue and visible condition were absent in the location judgment task in Experiment 1.

In Experiment 2, we addressed this discrepancy of using separate experiments for the semantic judgment task and the location judgment task in Experiment 1. Participants performed a semantic judgment task for the sequentially presented prime and target words and then performed a location judgment task for the target word in each trial. Similar to Experiment 1, the target visibility was manipulated by CFS and attention was manipulated by the cue, whereas the three (visible, congruent, and incongruent) conditions were randomly presented. The semantic judgment performance was $86 \pm 11.96 \%$ in the visible condition $\left(t_{(21)}=13.97, p<0.001\right)$, but it was at chance when the target was rendered invisible in both the congruent $\left(50.57 \pm 7.19 \%, t_{(21)}=\right.$ $0.37, p=0.715)$ and incongruent $\left(50.19 \pm 6.33 \%, t_{(21)}=0.140\right.$, $p=0.890)$ conditions.

The location judgment performance indicated that visibility was not different in the congruent and incongruent conditions. Specifically, participants occasionally detected the target location in both the congruent $\left(61.17 \pm 18.73 \%, t_{(21)}=2.8, p=0.011\right)$ and incongruent $\left(58.24 \pm 19.33 \%, t_{(21)}=2.0, p=0.059\right)$ conditions while participants reliably detected the target in the visible condition $\left(99.05 \%, t_{(21)}=164.59, p<0.001\right)$. The location judgment performance of the congruent condition significantly correlated with that of the incongruent condition across participants $(r=0.85, p<0.001$; Fig. 5A) without a significant difference in their average performance $\left(t_{(21)}=1.30, p=0.207\right)$. However, we did not find any correlation between the location judgment and semantic judgment performance in both the congruent $(r=0.14$, $p=0.545$; Fig. $5 B)$ and incongruent conditions $(r=-0.32$, $p=0.141$; Fig. $5 C$ ). These results demonstrate that semantic information was not consciously processed when the interocular suppression was incomplete as assessed by location detection.

\section{Discussion}

In the present study, we provide evidence that attention modulates the extent of unconscious semantic processing of invisible stimuli during CFS. The N400 modulation was absent when attention was given to the target location during CFS. However, the N400 modulation emerged when the location of attention was diverted away from the target words. In addition, the magnitude of the N400 modulation obtained from the incongruent condition was negatively correlated with the location judgment performance, suggesting that the more attention is withdrawn from the target location, the larger the N400 that is obtained. These results indicate that inattention can facilitate semantic processing of the invisible stimuli during CFS.

\section{The $\mathrm{N} 400$ as a tool for probing unconscious semantic processing}

The N400 is a sensitive tool for revealing semantic processing, even in the absence of any behavioral difference between related and unrelated target words (Luck et al., 1996; Heil et al., 2004; Vogel et al., 2005; Giesbrecht et al., 2007). We, however, addressed two methodological concerns. First, attention modulates unconscious semantic processing and, thus, the N400 component (Kiefer, 2007; Kutas and Federmeier, 2011). For example, Giesbrecht et al. (2007) conducted an attentional blink study. In this study, the participants judged semantic relatedness between Target 1 (T1) and Target 2 (T2) while the perceptual load of the $\mathrm{T} 1$ task was manipulated. The load of the T1 task was supposed to modulate the attentional resources available for the T2 task such that, if the load was low, attentional resources should be available for processing the T2 during the attentional blink. On the other hand, a high load should lead to depletion of attention resources for the T2 task; thus, semantic processing should not occur during the blink (Lavie, 1995; Lavie et al., 2004). Consistently, Giesbrecht et al. (2007) found that the N400 modulation was present when the perceptual load was low but disappeared when the load was high. According to this account, attention can modulate unconscious semantic processing if attentional resources available for the invisible target of the congruent and incongruent conditions are different. However, if the increased attention at the cued location facilitated the suppression of the invisible target as shown by the previous studies, there is no reason to assume that 
the suppressed target of the congruent condition would have been processed with more attention.

Second, masked priming procedures have been used to assess the extent of unconscious semantic processing, and they have provided behavioral (e.g., reaction times) as well as electrophysiological evidence for unconscious semantic processing (Kouider and Dehaene, 2007; for review, see Kiefer, 2007). In the masked priming procedures (Brown and Hagoort, 1993; Kiefer, 2002; Ortells et al., 2016), for example, a target word is followed by a prime with forward and backward masks and the participants make a judgment of the target (e.g., lexical judgment task) as fast and as accurately as possible. The behavioral evidence for unconscious semantic processing is that reaction times are faster when the masked prime and the visible target are semantically related than when they are unrelated. On account of these results, one may wonder whether we could have obtained similar behavioral evidence (reaction times) in addition to the N400. In the present study, however, the participants determined the semantic relatedness of the visible prime and the invisible target, which is different from the masked priming procedure where judgments can be made only for the visible target words. Therefore, it was not suitable to analyze reaction times for the invisible target of the present study; thus, we did not emphasize the speed of responses during the experiment either.

\section{Attention and unconscious semantic processing in interocular suppression}

We demonstrated that reduced interocular suppression due to inattention is a key factor for unconscious semantic processing during CFS. Considering a common sense assumption that attention facilitates information processing, this result is counterintuitive. However, we claim that the unconscious semantic processing due to inattention under interocular suppression is highly plausible. Based on the previous findings, reduced attention attenuates the strength of interocular suppression (Bahrami et al., 2007; Zhang et al., 2011; Brascamp and Blake, 2012; Ling and Blake, 2012) and semantic information is processed without attention (Luck et al., 1996; Vogel et al., 2005; Giesbrecht et al., 2007). Therefore, reduced attention to the location of the target word in the incongruent condition attenuated interocular suppression; thus, semantic information became available unconsciously. On the other hand, how attention operates on rival stimuli can also explain the absence of semantic processing in the congruent condition. When two stimuli were binocularly competing, attention modulated the adaptation aftereffect of the dominant stimulus but not of the suppressed stimulus, indicating that the effect of attention was manifested in only the visible stimulus (Jung and Chong, 2014). In the context of the present study, attention to the target location in the congruent condition should have strengthened the dominant CFS stimuli; thus, the target information should have been more suppressed. Therefore, fully engaged attention under interocular suppression prevents the visual system from processing the meaning of the suppressed target.

\section{Location uncertainty can facilitate unconscious processing in previous studies}

If we examine previous studies investigating unconscious semantic processing during interocular suppression, our results are the more plausible and even reconcile conflicting evidence. Specifically, in previous studies showing unconscious semantic processing during CFS (Costello et al., 2009; Bahrami et al., 2010; Yang and Yeh, 2011; Sklar et al., 2012; Zabelina et al., 2013), participants were unable to predict the location of a suppressed stimulus because the suppressed target was randomly presented, for exam- ple, to either above or below a fixation point (Costello et al., 2009; Sklar et al., 2012). However, in a study where the suppressed target word was presented in the middle of the display and thus attention was fully directed to the target, unconscious semantic analysis did not occur (Kang et al., 2011). Our finding suggests that location uncertainty of the suppressed stimuli in the former studies (Costello et al., 2009; Sklar et al., 2012) prevents spatial attention from fully operating in the location of the target (Palmer et al., 1993); thus, the semantic information under suppression could have been processed in the absence of awareness.

Our results can also be applied to other higher-level processing (e.g., object recognition or categorization) in the absence of awareness induced by interocular suppression. Numerous studies have shown that object information is processed without awareness during interocular suppression (Jiang et al., 2007; Yang et al., 2007; Zhou et al., 2010; Mudrik et al., 2011; Stein et al., 2011 a,b, 2012; Stewart et al., 2012). For instance, upright faces (Jiang et al., 2007; Zhou et al., 2010; Stein et al., 2011a, 2012), faces with direct gaze (Stein et al., 2011b; Yokoyama et al., 2013), or fearful faces (Yang et al., 2007; Stein et al., 2014) break suppression faster than inverted faces, faces with averted gaze, or neutral faces, respectively. In addition, incongruent scenes (e.g., soccer players dribbling a watermelon instead of a soccer ball) are detected faster than congruent scenes (Mudrik et al., 2011). One common ground of these studies is that participants could not predict the presented location of target stimuli (e.g., one of four quadrants) or its key features within a scene so that attention could have been directed anywhere. As a result, reduced attention to the target stimulus attenuated interocular suppression as in the incongruent condition of the current study.

\section{The Global Neuronal Workspace model and the extent of unconscious processing}

Our finding supports the Global Neuronal Workspace model that proposes two distinctive types of unconscious states (Dehaene et al., 2006; Kouider and Dehaene, 2007). In the subliminal state, information processing in higher processing stages does not occur because of weak sensory inputs. In the preconscious state, sensory information does not reach the conscious state with lack of top-down attentional amplification but that information is available for further processing. Here, we demonstrated that inattention could facilitate unconscious processing during interocular suppression. Specifically, strong suppression due to attention directed to the target location creates the subliminal state, making its sensory information too weak so that semantic processing does not occur. In contrast, if attention is diverted away from the target location, which weakens the rivalry suppression, the sensory information of the target gets stronger for further processing. Nevertheless, because attention is diverted away, the sensory information fails to receive attentional amplification to reach a conscious state, resulting in the preconscious state. This interpretation can also be applied to previous studies without contradiction (Costello et al., 2009; Bahrami et al., 2010; Kang et al., 2011; Yang and Yeh, 2011; Sklar et al., 2012; Zabelina et al., 2013).

\section{References}

Almeida J, Mahon BZ, Nakayama K, Caramazza A (2008) Unconscious processing dissociates along categorical lines. Proc Natl Acad Sci U S A 105: 15214-15218. CrossRef Medline

Bahrami B, Lavie N, Rees G (2007) Attentional load modulates responses of human primary visual cortex to invisible stimuli. Curr Biol 17:509-513. CrossRef Medline

Bahrami B, Vetter P, Spolaore E, Pagano S, Butterworth B, Rees G (2010) Unconscious numerical priming despite interocular suppression. Psychol Sci 21:224-233. CrossRef Medline 
Blake R (1988) Dichoptic reading: the role of meaning in binocular rivalry. Percept Psychophys 44:133-141. CrossRef Medline

Blake R, Tadin D, Sobel KV, Raissian TA, Chong SC (2006) Strength of early visual adaptation depends on visual awareness. Proc Natl Acad Sci U S A 103:4783-4788. CrossRef Medline

Brainard DH (1997) The Psychophysics Toolbox. Spat Vis 10:433-436. CrossRef Medline

Brascamp JW, Blake R (2012) Inattention abolishes binocular rivalry: perceptual evidence. Psychol Sci 23:1159-1167. CrossRef Medline

Brown C, Hagoort P (1993) The processing nature of the N400: evidence from masked priming. J Cogn Neurosci 5:34-44. CrossRef Medline

Costello P, Jiang Y, Baartman B, McGlennen K, He S (2009) Semantic and subword priming during binocular suppression. Conscious Cogn 18: 375-382. CrossRef Medline

Dehaene S, Changeux JP, Naccache L, Sackur J, Sergent C (2006) Conscious, preconscious, and subliminal processing: a testable taxonomy. Trends Cogn Sci 10:204-211. CrossRef Medline

Delorme A, Makeig S (2004) EEGLAB: an open source toolbox for analysis of single-trial EEG dynamics including independent component analysis. J Neurosci Methods 134:9-21. CrossRef Medline

Gayet S, Van der Stigchel S, Paffen CL (2014) Breaking continuous flash suppression: competing for consciousness on the pre-semantic battlefield. Front Psychol 5:460. CrossRef Medline

Giesbrecht B, Sy JL, Elliott JC (2007) Electrophysiological evidence for both perceptual and postperceptual selection during the attentional blink. J Cogn Neurosci 19:2005-2018. CrossRef Medline

Heil M, Rolke B, Pecchinenda A (2004) Automatic semantic activation is no myth: semantic context effects on the N400 in the letter-search task in the absence of response time effects. Psychol Sci 15:852-857. CrossRef Medline

Hesselmann G, Darcy N, Sterzer P, Knops A (2015) Exploring the boundary conditions of unconscious numerical priming effects with continuous flash suppression. Conscious Cogn 31:60-72. CrossRef Medline

Jiang Y, Costello P, He S (2007) Processing of invisible stimuli: advantage of upright faces and recognizable words in overcoming interocular suppression. Psychol Sci 18:349-355. CrossRef Medline

Jung Y, Chong SC (2014) Effects of attention on visible and invisible adapters. Perception 43:549-568. CrossRef Medline

Kang MS, Blake R, Woodman GF (2011) Semantic analysis does not occur in the absence of awareness induced by interocular suppression. J Neurosci 31:13535-13545. CrossRef Medline

Kiefer M (2002) The N400 is modulated by unconsciously perceived masked words: further evidence for an automatic spreading activation account of N400 priming effects. Cogn Brain Res 13:27-39. CrossRef Medline

Kiefer M (2007) Top-down modulation of unconscious 'automatic' processes: a gating framework. Adv Cogn Psychol 3:289-306. CrossRef Medline

Kim CY, Blake R (2005) Psychophysical magic: rendering the visible invisible.' Trends Cogn Sci 9:381-388. CrossRef Medline

Kouider S, Dehaene S (2007) Levels of processing during non-conscious perception: a critical review of visual masking. Philos Trans R Soc Lond B Biol Sci 362:857-875. CrossRef Medline

Kutas M, Federmeier KD (2011) Thirty years and counting: finding meaning in the N400 component of the event-related brain potential (ERP). Annu Rev Psychol 62:621-647. CrossRef Medline

Kutas M, Hillyard SA (1980) Reading senseless sentences: brain potentials reflect semantic incongruity. Science 207:203-205. CrossRef Medline

Lavie N (1995) Perceptual load as a necessary condition for selective attention. J Exp Psychol Hum 21:451-468. CrossRef Medline

Lavie N, Hirst A, de Fockert JW, Viding E (2004) Load theory of selective attention and cognitive control. J Exp Psychol Gen 133:339-354. CrossRef Medline

Lin Z, He S (2009) Seeing the invisible: the scope and limits of unconscious processing in binocular rivalry. Prog Neurobiol 87:195-211. CrossRef Medline

Ling S, Blake R (2012) Normalization regulates competition for visual awareness. Neuron 75:531-540. CrossRef Medline

Luck SJ (2014) An introduction to the event-related potential technique, Ed 2. Cambridge, MA: Massachusetts Institute of Technology.

Luck SJ, Vogel EK, Shapiro KL (1996) Word meanings can be accessed but not reported during the attentional blink. Nature 383:616-618. CrossRef Medline

Mudrik L, Breska A, Lamy D, Deouell LY (2011) Integration without aware- ness: expanding the limits of unconscious processing. Psychol Sci 22: 764-770. CrossRef Medline

Ortells JJ, Kiefer M, Castillo A, Megías M, Morillas A (2016) The semantic origin of unconscious priming: behavioral and event-related potential evidence during category congruency priming from strongly and weakly related masked words. Cognition 146:143-157. CrossRef Medline

Palmer J, Ames CT, Lindsey DT (1993) Measuring the effect of attention on simple visual search. J Exp Psychol Hum Percept Perform 19:108-130. CrossRef Medline

Park T (2004) Investigation of association frequency and imagery value of Korean words. Korean J Exp Psychol 16:237-260.

PelliDG (1997) TheVideoToolbox software for visual psychophysics: transforming numbers into movies. Spat Vis 10:437-442. CrossRef Medline

Pratte MS, Rouder JN (2009) A task-difficulty artifact in subliminal priming. Atten Percept Psychophys 71:1276-1283. CrossRef Medline

Sklar AY, Levy N, Goldstein A, Mandel R, Maril A, Hassin RR (2012) Reading and doing arithmetic nonconsciously. Proc Natl Acad Sci U S A 109: 19614-19619. CrossRef Medline

Stein T, Hebart MN, Sterzer P (2011a) Breaking continuous flash suppression: a new measure of unconscious processing during interocular suppression? Front Hum Neurosci 5:167. CrossRef Medline

Stein T, Senju A, Peelen MV, Sterzer P (2011b) Eye contact facilitates awareness of faces during interocular suppression. Cognition 119:307-311. CrossRef Medline

Stein T, Sterzer P, Peelen MV (2012) Privileged detection of conspecifics: evidence from inversion effects during continuous flash suppression. Cognition 125:64-79. CrossRef Medline

Stein T, Seymour K, Hebart MN, Sterzer P (2014) Rapid fear detection relies on high spatial frequencies. Psychol Sci 25:566-574. CrossRef Medline

Sterzer P, Stein T, Ludwig K, Rothkirch M, Hesselmann G (2014) Neural processing of visual information under interocular suppression: a critical review. Front Psychol 5:453. CrossRef Medline

Stewart LH, Ajina S, Getov S, Bahrami B, Todorov A, Rees G (2012) Unconscious evaluation of faces on social dimensions. J Exp Psychol Gen 141: 715-727. CrossRef Medline

Swaab TY, Ledoux K, Camblin CC, Boudewyn M (2012) ERPs and language processing. In: Oxford handbook of event-related potential components, Chap 14 (Luck SJ, Kappenman ES, eds), pp 397-440. New York: Oxford UP.

Tsuchiya N, Koch C (2005) Continuous flash suppression reduces negative afterimages. Nat Neurosci 8:1096-1101. CrossRef Medline

Vogel EK, Woodman GF, Luck SJ (2005) Pushing around the locus of selection: evidence for the flexible-selection hypothesis. J Cogn Neurosci 17: 1907-1922. CrossRef Medline

Wiesenfelder H, Blake R (1990) The neural site of binocular rivalry relative to the analysis of motion in the human visual system. J Neurosci 10: 3880-3888. Medline

Yang E, Blake R (2012) Deconstructing continuous flash suppression. J Vis 12:8. CrossRef Medline

Yang E, Zald DH, Blake R (2007) Fearful expressions gain preferential access to awareness during continuous flash suppression. Emotion 7:882-886. CrossRef Medline

Yang E, Brascamp J, Kang MS, Blake R (2014) On the use of continuous flash suppression for the study of visual processing outside of awareness. Front Psychol 5:724. CrossRef Medline

Yang YH, Yeh SL (2011) Accessing the meaning of invisible words. Conscious Cogn 20:223-233. CrossRef Medline

Yokoyama T, Noguchi Y, Kita S (2013) Unconscious processing of direct gaze: evidence from an ERP study. Neuropsychologia 51:1161-1168. CrossRef Medline

Zabelina DL, Guzman-Martinez E, Ortega L, Grabowecky M, Suzuki S, Beeman M (2013) Suppressed semantic information accelerates analytic problem solving. Psychon Bull Rev 20:581-585. CrossRef Medline

Zhang P, Jamison K, Engel S, He B, He S (2011) Binocular rivalry requires visual attention. Neuron 71:362-369. CrossRef Medline

Zhou G, Zhang L, Liu J, Yang J, Qu Z (2010) Specificity of face processing without awareness. Conscious Cogn 19:408-412. CrossRef Medline

Zimba LD, Blake R (1983) Binocular rivalry and semantic processing: out of sight, out of mind. J Exp Psychol Hum Percept Perform 9:807-815. CrossRef Medline 